\title{
35 ANS D'ÉLECTIONS EN CATALOGNE: DE L'AUTONOMISME À L'INDÉPENDANTISME
}

Joan Marcet, Lucia Medina, Robert Liñeira, traduit de l'espagnol par Jacques

Fontaine

ARPoS | « Pôle Sud »

2014/1 n 40 | pages 81 à 97

ISSN 1262-1676

ISBN 9782918036180

Article disponible en ligne à l'adresse :

https://www.cairn.info/revue-pole-sud-2014-1-page-81.htm

Distribution électronique Cairn.info pour ARPoS.

(C) ARPoS. Tous droits réservés pour tous pays.

La reproduction ou représentation de cet article, notamment par photocopie, n'est autorisée que dans les limites des conditions générales d'utilisation du site ou, le cas échéant, des conditions générales de la licence souscrite par votre établissement. Toute autre reproduction ou représentation, en tout ou partie, sous quelque forme et de quelque manière que ce soit, est interdite sauf accord préalable et écrit de l'éditeur, en dehors des cas prévus par la législation en vigueur en France. Il est précisé que son stockage dans une base de données est également interdit. 


\title{
35 ans d'élections en Catalogne : DE L'AUTONOMISME À L'INDÉPENDANTISME
}

\author{
Joan Marcet \\ joan.marcet@uab.cat \\ Lucia Medina \\ medinall@diba.cat \\ Robert Liñeira \\ robert.lineira@uab.cat
}

Université autonome de Barcelone

Institut de ciències polítiques i socials

Traduit del'espagnol par Jacques Fontaine

\section{RÉsumé / ABstract}

Depuislespremièresélections démocratiquesen Espagneen juin 1977 jusqu'ànosjours, onze élections générales (au Congrès des D éputés et au Sénat) et dix élections au Parlement de Catalogne se sont déroulées en C atalogne. T out au long de ces 35 ans, le comportement électoral des citoyens catalans s'est signalé par deux singularités : une disparite frappante dans la participation en fonction du type de scrutin et un vote le plus souvent différent suivant qu'il s'agit d'élire le G ouvernement espagnol ou celui de C atalogne lors desélections législatives concernant l'un ou l'autre. La confrontation électorale en Catalogne a tourné comme à l'accoutumée autour de deux axes politico-idéologiques. D'un côté, la dimension gauche-droite et, de l'autre, le pivot qu'on appelle nationaliste, défini à partir du sentiment d'appartenance vis-à-vis de l'Espagne ou de la C atalogne ainsi que par ce que l'on considère comme étant la meilleure forme d'insertion de la Catalogne au sein de l'É tat espagnol. Le changement le plus significatif intervenu au cours de ces trois dernières années a été la progression de l'option en faveur d'une C atalogne indépendante de l'Espagne, laquelle est parvenue à se placer à la première place des préférences des citoyens catalans.

Since celebrating the first democratic elections in Spain in June of 1997, Catalonia has had eleven of its own general elections ( Congress of Representatives and the Senate) and 10 elections for the Catalonian Parliament. Throughout these 35 years the voting behavior of the citizens of Catalonia has been characterized by two singular facts : a notable difference of electoral turnout according to the electoral call and a largely different vote as they were choosing the Government of Spain or Catalonia, through respective parliamentary elections. The ecctoral confrontation in Catalonia has traditionally moved into two political-ideological axes. $0 \mathrm{n}$ one side is theleft-right dimension and on the other side is the national axis, configured from the feeling of belonging to Spain or Catalonia and the consideration of the best way to fit Catalonia into the Spanish State. The most significant change which has developed in the past three years has been the growing support for the option of an independent Catalonia in Spain, something which has becomea top preferencefor $\mathrm{C}$ atalonian citizens.

\section{MOTS-CLÉS / KEYWORDS}

Comportement électoral, élections, indépendantisme, nationalisme, partis politiques, système de partis

Elections, electoral behavior, independence, nationalism, party system, political parties 


\section{UN TERRITOIRE, DEUX ÉLECTIONS : QUELQUES PAR- TICULARITÉS DU COMPORTEMENT ÉLECTORAL EN CATALOGNE}

Depuis la tenue en juin 1977 des premières élections démocratiques en Espagneet jusqu'à maintenant, se sont déroulées en C atalogne onzeélections générales (au Congrès des D éputés et au Sénat) et dix élections au Parlement de Catalogne ${ }^{1}$. Au cours de ces trente cinq dernières années, le comportement électoral des citoyens de $C$ atalogne s'est fait remarquer par deux faits singuliers: d'une part, s'agissant de la participation électorale une différence considérable en fonction de la nature du scrutin et d'autre part, des résultats en suffrages très différents suivant qu'il s'agit d'élire le Gouvernement espagnol ou celui de Catalogne lors de leurs élections parlementaires respectives.

Lors des élections générales, la participation en C atalogne se situe d'habitudeautour des $70 \%$ avec un maximum de $80,8 \%$ en 1982 et un minimum de $65,2 \%$ au cours des dernières élections de 2011 . Au contraire, pour les élections autonomiques au Parlement deC atalogne, la participation en moyenne gravite autour des $60 \%$ avec un minimum de $54,90 \%$ en 1992 et un maximum historique de $67,80 \%$ lors du dernier scrutin en novembre 2012. Cette différence habituelle de participation électorale de quasiment dix points entre les deux types d'élections a été largement abordée par la littérature académique à travers diverses analyses sur la dénommée « abstention différentielle 》 (Font, Contreras \& Rico, 1998 ; Riba, 1995, 2000 ; Vallès, 2008, 2009). À travers le comportement homogène de toutes les $C$ ommunautés Autonomes dans lesquelles on constate toujours une participation plus importante pour les élections générales que pour les élections autonomiques, des études abordant les années 80-90 ont mis l'accent sur la singularité catalane concernant la participation en fonction du type d'élection. Au cours de la période 19821993, la participation en moyennelors des élections autonomiques est même inférieure à celle constatée pour les élections municipales (Riba, 2000). Les études en question mettent en avant comme principales causes de cette abstention différentielle (Pallarés \& Font, 1995) l'absence d'identification par rapport aux institutions propres à la C atalogne, l'origine territoriale d'un secteur de la population catalane, provenant del'immigration intérieuredu siècle dernier et adhérant bien peu au sentiment national catalan ou bien encore l'existence de problèmes concernant l'offre politique.

Des travaux plus récents (Vallès, 2009) pointent et rajoutent d'autres facteurs explicatifs, plus en rapport avec le fait de considérer les élections autonomiquescommeetant des « élections desecond ordre» ou bien avecl'enracinement social moins important de la population des grands centres urbains étant

\footnotetext{
1. Pour cette analyse des élections en Catalogne nous avons écarté les deux niveaux électoraux comportant une logique politique différente : les élections municipales qui ont en général une logique locale difficilement extrapolable au niveau territorial autonomique et les élections au Parlement européen qui, en dépit des lectures et des interprétations de politique intérieure propagées dans chaque pays, entretiennent égal ement leur proprelogique au regard dela construction européenne.
} 
donné que le différentiel de participation se produit de manière semblable dans les conurbations de Barcelone et de $\mathrm{M}$ adrid.

Le fait de considérer les élections autonomiques comme moins importantes que les élections général es a été une constante dansl'opinion publique catalane jusqu'en 2011. Jusqu'à cette date, ces dernières étaient considérées comme plus importantes par un pourcentage de l'opinion publique oscillant entre 34 et $44 \%$ tandis que la proportion de celle estimant que c'était plutôt les élections autonomiques se situait entre 20 et 26\% (une moyenne de $15 \%$ donnait à toutes les élections la même importance) ${ }^{2}$. D ans les deux derniers sondages d'opinion réalisés par I'ICPS et correspondants à 2012 et 2013, les élections autonomiques apparaissent pour la première fois comme plus importantes que les élections générales. Ce changement repéré dans les sondages coïncide avec un changement de comportement électoral étant donné que lors des dernières élections autonomiques de novembre 2012, on assiste pour la première fois à une participation supérieure à celle des élections générales qui venaient tout juste de les précéder en novembre 2011.

Le débat politique, polarisé davantage sur le positionnement de la Catalogne par rapport à l'Espagne plutôt que sur les effets de la crise économique et les politiques conduites pour y faire face, a contribué sans nul doute à cette participation plus importante. Pareillement le prolongement tout au long de 2013 du débat focalisé, politiquement et médiatiquement, sur le fond et la forme de la relation C atalogne-Espagne et la possibilité d'un referendum sur l'indépendance dela Catalogne, font prendreconscienceàl'opinion publique del'importance plus grande des élections autonomiques catalanes ${ }^{3}$.

En même temps, à l'explication traditionnelle de l'abstention différentielle due à des variables de caractère « identitaire-national », lesétudes comparées effectuées sur le comportement électoral des grandes conurbations permettent d'ajouter d'autres facteurs explicatifs comme l'existence d'une certaine culture « néo-urbaine» (Vallès, 2009) ou la progression de phénomènes comme la désaffection politique (Vallès, 2008 ; Blanco \& Mas, 2008). Dans ce cas à nouveau, le débat politique suscité par la convocation aux élections de novembre 2012 expliquerait la progression de la participation dans les secteurs urbains de $C$ atalogne, habituellement plus abstentionnistes.

Le second élément qui caractérise les élections en C atalogne, ainsi que nous l'avons noté au début, se rapporte au comportement électoral diversifié descatalans suivant qu'il s'agit d'électionsau C ongrès des Députés d'Espagne ou d'élections au Parlement de Catalogne. Ce comportement électoral différencié, que l'on a appelé « vote dual » (M ontero \& Font, 1989, 1991; Riba, 1995, 2000) a fait que jusqu'aux élections de 2011 les socialistes aient toujours obtenu en Catalogne la majorité des suffrages et sièges aux élections générales et, par contre, pour lesélections au Parlement de Catalognela victoire

2. Cf. les données fournies par les « Sondeos de 0 pinión Catalunya » réalisés par l'Institut de Sciences Politiques et Sociales (ICPS) de 1989 ànos jours.

3.Cf. le « Sondeo deO pinión Catalunya » de 2013 effectué parl'ICPS. Les différents baromètres d'opinion réalisés au cours de 2013 par leC entre d'Études d'O pinion de la Generalitat de Catalogneapportent des résultats similaires. 
ait été toujours dévolue à la formation de centre-droit de tendance nationaliste Convergència i U nió (CiU $)^{4}$.

Les graphiques 1 et 2 indiquent cette différence de résultats électoraux en Catalogne en fonction du type d'élection : au Congrès des Députés ou au Parlement de C atalogne. Aux élections nationales le PSC s'est positionné jusqu'en 2008 comme la première force politique avec au début 28,6 \% des suffrages lors des premières élections, puis en dépassant les $45 \%$ aux élections de 1982 et 2008, ayant comme principal compétiteur la coalition nationaliste deCiU, parti exclusivement catalan, et, dans une moindre mesure, le Partido Popular en compétition, sur le plan national, avec les socialistes depuis les années 80 ànos jours. Au contraireauxélections autonomiques au Parlement de $\mathrm{C}$ atalogne, $\mathrm{CiU}$ se maintient en tant que première force politique à partir de 1980 avec $27,80 \%$ des suffrages, obtenant trois majorités absolues dans les élections suivantes (1984, 1988 et 1992), amorçant un progressif déclin en nombre de votes entre 1995 et 2006, recouvrant son hégémonie en grande partie lors des élections de 2010, pour à nouveau régresser légèrement au cours des dernières élections de 2012. Aux élections autonomiques le Parti Socialiste s'est toujours vu relégué à la deuxième place, à l'exception notable en nombre de suffrages exprimés, des élections de 1999 et de 2003 à partir desquelles s'enclenche un net déclin au point de le situer aux élections de 2012 à peu de distance des trois autres partis du tableau politique catalan.

\section{Graphique 1. Résultats électoraux aux élections} au Congrès des Députés en Catalogne

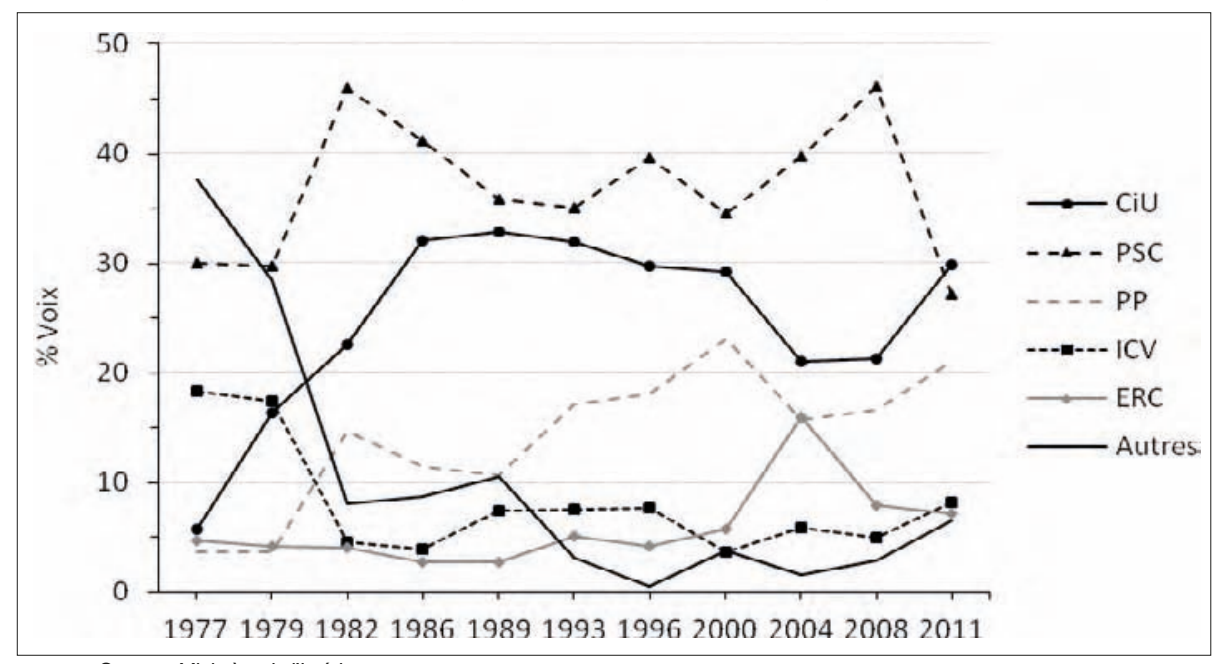

Source : Ministère de l'Intérieur

$\overline{4 . A u x e ́ l e c t i o n s ~ d e ~} 1999$ et 2003 quand bien mêmelescandidaturessocialistes eurent remporté un plus grand nombre de suffrages, la répartition spéciale des sièges en quatre districts ou circonscriptionsélectorales a octroyéun plus grand nombre desièges à CiU. 
Graphique 2. Résultats électoraux aux élections au Parlement de Catalogne

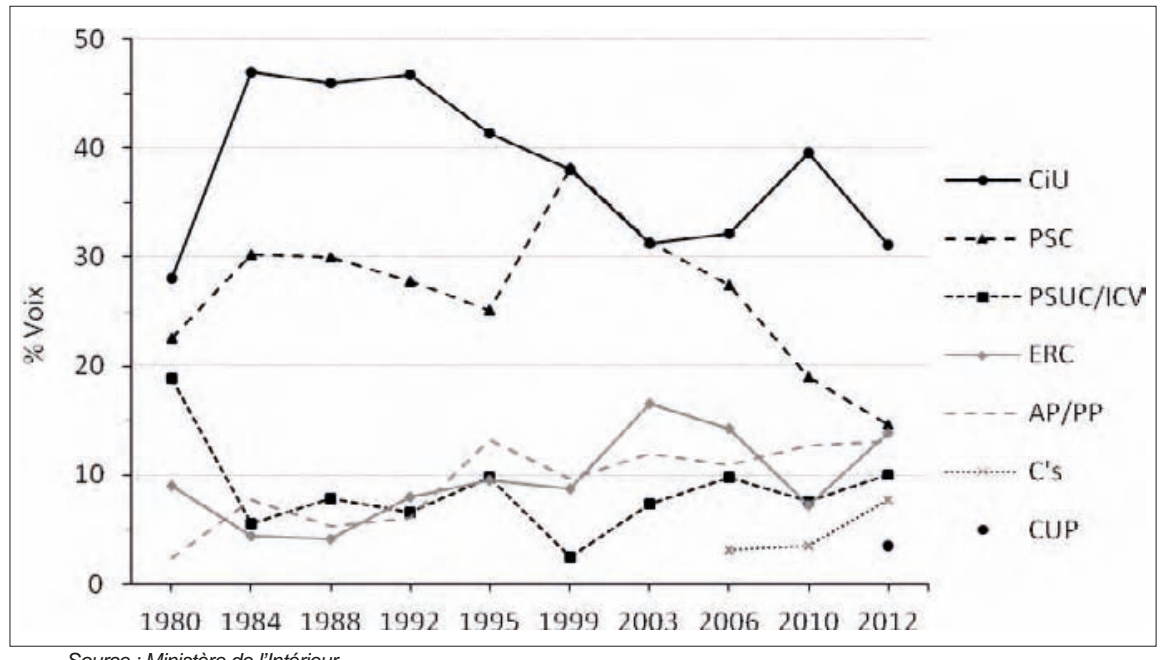

Source : Ministère de l'Intérieur

Ce comportement électoral dual peut largement s'expliquer par la pré sence en Catalogne d'un système de partis particulier dans lequel la compétition entre eux est plutôt pluraliste - différent de celui existant à l'échelle nationale - et principalement animé par des partis sans dimension nationale qui recherchent à travers divers cheminements à influer sur le cours de la politique gouvernementale ( $M$ arcet, 2011). Ces graphiques de résultats électorauxillustrent la compétition la plus importante et la plus pluraliste qu'ait vécue la Catalogne, contrastant avec celle existante en Espagne jusqu'à présent, monopolisée par un affrontement entre le PSOE et le PP, alternant au pouvoir de 1982 à nos jours. La concentration électorale en Espagne s'est progressivement accrue de la fin des années 80 aux élections de 2008, année où elle atteint quasiment $84 \% 5$ et la concentration parlementaire, 92\% la même année. Au contraire, en Catalogne, la concentration électorale aux élections nationales oscille entre 60 et $70 \%$ et, lors des élections au Parlement Catalan, elle n'a cessé de diminuer à partir de la fin des années 90 pour atteindre le modeste chiffre de $45 \%$ aux dernières élections de 2012. Les résultats des dernières élections aussi bien nationales qu'autonomiques, en 2011 et 2012 respectivement, malgréle fait de constituer un changement remarquable par rapport à la série d'élections précédentes ${ }^{6}$, viennent corroborer la présence d'un système de partis spécifique, marqué par une plus grande proximité des différentes forces électorales.

5. Aux dernièresélections nationales, en 2011, la concentration électoralea reculépratiquement de dix points (jusqu' aux 73,5\%), ceci dû principalement à l'effondrement électoral du PSOE. 6. Lors de cesélections, CiU décroche pour la première fois la victoireà des élections nationales (2011) et auxélections autonomiques de 2012, bien que lePSC se maintienneen tant que deuxième force électorale, il perd cette position au niveau parlementaire dans la mesure où il voit son nombre de sièges obtenus dépassé par Esquerra Republicana de Catalunya. 
O utre cet élément explicatif du système catalan des partis, dans les multiplestravaux analysant le comportement dichotomique del'électorat catal an, on relève d'autres motifs mis en avant par les citoyens de C atalogne pour justifier leur changement de comportement en fonction du type d'élections. À savoir : l'identité catalane, la meilleure défense de leurs interêts en regard des choix offerts, l'importance des axes de confrontation politico-idéologiques selon le type d'élection, en passant enfin par la recherche de nécessaires et pertinents equilibre institutionnel et répartition de pouvoirs (Colomer, 1991 ; M ontero \& Font, 1991 ; Riba, 2000).

Le fait de considérer le PSC comme le parti le mieux à même de représenter et défendre les intérêts des citoyens de C atalogne au niveau national a étéévident jusqu'en 2011 ainsi que cela ressort des sondages d'opinion politique réalises par I'ICPS. En revanche toujours selon ces sondages, les mêmes citoyens ont constamment estimé que c'est la coalition nationaliste de CiU qui incarne le mieux leurs intérêts dans le milieu politique catalan. C'est seulement au milieu du mandat du Gouvernement tripartite des gauches à la Generalitat de Cataluña (2003-2008), dirigé alors par le PSC allié à ERC et ICV, queles citoyens estiment que les socialistes sont les meilleurs défenseurs également de leurs intérêts dans la politique catalane?.

L'affrontement électoral en Catalogne a tourné traditionnellement autour de deux axes politico-idéologiques. D'un côté, la dimension gauche-droite qui, jointe à l'appartenance à la classe sociale ou au taux de religiosité, est considéré comme un élément fondamental du comportement électoral ${ }^{8}$. De l'autre, le dénommé axe nationaliste, dessinéà partir du sentiment d'appartenance par rapport à l'Espagne ou à la C atalogne, et par l'estimation de ce que doit être la forme la plus souhaitable de positionnement de la C atalogne par rapport à l'État espagnol, ce qui conduit de l'option en faveur d'une Espagne unitaire et centralisée jusqu'au souhait d'une Catalogne indépendante de l'État espagnol. L'entrecroisement de ces deux axes dans la compétition électorale trace un espace bidimensionnel dans lequel peut prendre place l'électorat de $C$ atalognesuivant letype d'élection et lasituation historique analysée (Bartomeus \& M edina, 2011). L'existence de ce double axe de compétition a généréla théorie du vote dichotomique au sein de la déjà très ample littérature académique sur cette question ( M ontero \& Font, 1989, 1991; Pallarés \& Font, 1995 ; Riba, 1995 \& 2000 ; M olas \& Bartomeus, 1998, 1999).

Une étude récente de l'ICPS montre clairement pour ce qui concerne les relations $C$ atalogne et Espagne comment les preférences des catalans ont évolué à partir de la fin des années 80 jusqu'à nos jours (Liñeira, 2013). Depuis l'attribution à la C atalogne du statut de Communauté Autonome, ce statut est restée l'option préféree des catalans jusqu'en 2011 avec un soutien variable partant de $40 \%$ au début des années 90 , progressant presque à $60 \%$ en 2007 et amorçant à partir de cette même annee une chute pour atteindre $25 \%$ en 2013. L'option en faveur del'instauration d'un État àl'intérieur d'une

7. Cf. les sondages d'opinion politique catalane effectués par l'ICPS au cours de cette période. 8. Pour appréhender l'incidence de ces facteurs au niveau européen, cf. Thomassen (2005) et danslecas espagnol (Torcal \& M edina, 2007). 
Espagne exclusivement fédérale a connu un certain succès entre 2002 et 2008, période au cours de laquelle on a assisté au pari d'une réforme du Statut d'Autonomie de la Catalogne à l'instigation du Gouvernement tripartite dirigé par les socialistes. Le changement le plus significatif, c'est la progression du choix en faveur d'une Catalogne indépendante de l'Espagne. Cette éventualité habituellement emportait l'adhésion d'environ 20\% des citoyens de C atalogne mais, à partir de 2011, elles'imposeà la première place de leurs préférences franchissant les $42 \%$ en $2013^{\circ}$. C ette évolution du choix des citoyens catalans concernant les rapports $C$ atalogne-E spagne va dans le même sens que la mutation du sentiment identitaire ; s'agissant de celle-ci, depuis le début de la deuxième décennie du Xxle siècle, on a assisté à la progression du pourcentage des citoyens se considérant uniquement catalans (24\%) ou plus catalans qu'espagnols (27\%), la part de ceux ayant un sentiment partagé, autant catalans qu'espagnols ( $35 \%$ ) ${ }^{10}$, demeurant encore majoritaire.

L'éventualité de la tenue d'un référendum en Catalogne sur la question de son indépendance par rapport à l'Espagne s'est imposée comme le sujet principal dans l'agenda politique catalan et a suscité une abondante littérature, dans les médias, dans les milieux politiqueet, dans une moindremesure, académique. D ans cette dernière catégorie, on a assisté à la publication de certaines études quantitatives reposant sur les données offertes à l'heure actuelle par les sondages et les enquêtes d'opinion non sans que ceci soulève quelque controverse quant aux méthodes employées pour attribuer aux deux positions - favorables ou hostiles àl'indépendance catalane - le pourcentage d'indécis ou de ceux qui, pour l'instant, envisagent de ne pas participer à la consultation ${ }^{11}$.

Cet ensemble de facteurs permet d'expliquer le changement électoral intervenu lors des consultations les plus récentes en 2011 pour élire le Congrès des Députés d'Espagne et en 2012 pour l'élection du Parlement de Catalogne. La poursuite, en 2012 et 2013 en particulier, du débat identitaire au premier plan politique et médiatique, contribueà faire de celui-ci l'axemajeur de la confrontation politico-électorale, si ce n'est l'élément unique de débats et controverses. C e fait semble augurer un profond changement dans la carte électorale de la Catalogne au bénéfice d'options politiques défendant plus clairement des positions antagonistes et au détriment des partis politiques traditionnels qui ont animé jusqu'à maintenant la scène politique catalane. Les sondages d'opinion réalisés tout au long de 2013 laissent présager, face à l'éventualité de nouvelles élections au Parlement de C atalogne, une victoire d' Esquerra Republicana de Catalunya (ERC) incarnant l'option la plus fran-

9. Les données peuvent être consultées dans l'étude évoquée supra et publiée par I'ICPS ( L iñeira, 2013). Nous avons trouvé des chiffres similaires dans les baromètres récents établis par le Centre d'Études d'O pinion de la G eneralitat ( Cf. les trois vagues du baromètre 2013)

10. Les pourcentages indiqués correspondent au Sondage de I'ICPS en 2013. N ous avons trouvé des chiffres semblables à ceux des baromètres du $\mathrm{CEO}$ déjà évoqués.

11. Sur cette question on peut s'en référer aussi bien au document officiel (Cf. Le premier rapport du « Consell Assessor per a la T ransició N acional » de la Generalitat) qu'à certains travaux sur leshypothèses de participation et de résultats ( M uñoz, Guinjoan \& Vilaregut, 2010 ; Liñeira, 2013). 
chement indépendantiste ainsi qu'une progression très importante de Ciutadans-Partido por la Ciudadanía, principal défenseur du statu quo actuel et par conséquent du maintien de la C atalogne au sein de l'Espagne. Le parti jusqu'alors hégémonique - qui a gouverné la Catalogne durant 27 des 34 années d'autonomie catalane - CiU se verrait ainsi relégué en deuxième place malgré le virage indépendantiste qu'il a emprunté de façon majoritaire mais non unanime à partir de 2011. Pour leur part, les social istes du PSC se trouveraient cantonnés en quatrième position, en compétition étroite avec la gauche écolo-socialiste (ICV) et avec lePP. Assurément, aussi bien CiU que le PSC et le PP paient les pots cassés de leur passage ou séjour prolongé au Gouvernement en Catalogneou en Espagne ${ }^{12}$.

\section{UN SYSTÈME DE PARTIS SPÉCIFIQUE ET SINGULIER}

Comme nous l'avons déjà signalé, les caractéristiques du comportement électoral descitoyens catalansont contribuéàlaformation d'un systèmedepartis spécifique où coexistent des partis de dimension nationale et non nationale, entrelesquels se dispute une compétition des plus pluralistes.

Le déroulement en $C$ atalogne des premières élections autonomiques en 1980, après le rétablissement de la Généralité, confirme l'émergence de ce système de partis appeléà franchir plusieurs étapes. La première débute en 1980 et perdure jusqu'en 1992. Au cours de cette période, CiU se constitue une solide base électorale à partir de la mobilisation du vote catalaniste et du centre, lui permettant d'obtenir facilement des majorités de gouvernement, ce à quoi contribuent également les dynamiques abordées précédemment à savoir le vote dichotomique et l'abstention différentielle.

C ependant les élections au Parlement catalan de 1992 amorcent le début d'une étape nouvelle, caractérisée par l'affaissement de cette hégémonie. La coalition nationaliste commence à donner des signes d'usure après quasiment 15 ans de gouvernement, ce qui se traduit au travers des résultats obtenus aux élections autonomiques de 1995 au cours desquelles ellepassede 70 à 60 sièges. Q ui plus est, lors de ces mêmes élections se produit une avance importante du PP tout comme une remarquable progression d'ERC et d'I niciativa.

Les élections autonomiques d'automne 1999 inaugurent la troisièmeétape du systèmedepartiscatalan. Á lalassitudedueàtant d'années degouvernement $\mathrm{CiU}$ s'ajoute la défiance suscitée par le pacte de soutien mutuel entre ce parti et le PP pour assurer leur gouvernabilite aussi bien en C atalogne qu'au niveau de toutel'Espagne ${ }^{13}$. Dans de larges pans de l'électorat se répand alors le sentiment qu'il y a nécessité d'un changement politiquequi, aux yeux de beaucoup, s'incarne dans la figure de Pasqual Maragall (Castro, 2011). Aux élections de 1999, avec M aragall comme candidat à la présidence de la Généralité, le PSC recueille plus de suffrages que $\mathrm{CiU}$ mais ne parvient pas à obtenir un nombre de sièges suffisant pour occuper le gouvernement.

12. Cf. comme démonstration de cette tendanceles Baromètres du CEO de 2013.

13. On se souvient que les deux formations se trouvent en minorité dans leurs gouvernements respectifs. 
L'alternance politique n'a pas encore lieu mais au cours des mois suivants et, surtout à partir du triomphe du PP lors des élections générales de 2000, la tension s'accroit entre le nationalisme catalan et le gouvernement Aznar. Les opinions publiques catalane et espagnole se polarisent autour de la question territoriale; au terrain déjà prêt au changement, établi par les élections autonomiques de 1999, s'ajoutent les revendications en faveur d'une auto-gouvernance plus grande et les critiques àl'encontre du centralisme de M adrid.

La tenue des élections autonomiques le 16 novembre 2003 culmine avec ce processus de changement rendant l'alternance possible. A partir de cette date s'amorce une quatrième phase du système qui se prolongera jusqu'en 2010. Durant cette période, les socialistes catalans perdent leur élan électoral tandis que les écolo-socialistes, mais surtout la formation républicaine, connaissent une avancée importante par rapport aux résultats obtenus par eux aux élections de 1999, situations qui permettent de remplacer un gouvernement de centre-droit nationaliste par une succession de gouvernements de coalition PSC, ERC et ICV ${ }^{14}$.

Cette quatrième étape du système de partis se déroule de manière quelque peu tumultueuse en raison de problèmes internes à la coalition gouvernementale, de polémiques extérieures et de la difficulté à mettre en application le pacte ERC et PSC. La cohabitation au sein de la coalition s'avère compliquée parce qu'il existe des antagonismes identitaires importants, en particulier en ce qui concerne ces deux derniers alliés au gouvernement. Également l'élaboration d'un nouveau statut d'autonomie pour la Catalogne, dont le déploiement est rempli d'obstacles et de tensions, contribue à un accroissement de la polarisation identitaire et à l'émergence de nouveaux acteurs politiques, comme par exemple Ciudadanosel partido de la Ciudadanía (C's), force politique toute récente qui, au départ en tant que plateforme citoyenne, se revendique en tant que parti constitutionnaliste, non nationaliste et de centre-gauche.

Enfin, les élections autonomiques de 2010 entament une nouvelle phase, encore inachevée, dans le système de partis en Catalogne. Les élections de 2010 corroborent le redressement politique et électoral de CiU ${ }^{15}$. D e prime abord, il pourrait sembler que ce parti recouvre ainsi sa position de départ, en tant que parti hégémonique au sein d'une coalition nationaliste mais les résultats qu'il obtient lors des élections autonomiques anticipées en 2012, tempèrent cetteimpression ${ }^{16}$.

14. A près les élections au Parlement de C atalogne de 2003, on assiste précisément à la première formation d'un gouvernement tripartite, ce qui se reproduira trois ans plus tard après la tenue desélections autonomiques de 2006.

15. S'agissant des autres partis, PSC et ERC perdent une part importante de leurs électeurs, ICV parvient à se maintenir tandis quele PP améliore ses performances.

16. Les élections 2012 furent atypiques à plusieurs titres. En premier lieu parce qu'elles furent anticipées de deux ans avant la fin normale de la législature. En deuxième lieu, parce qu'on assiste à une augmentation importante de la participation électorale. Et en troisièmelieu parce qu'elles coïncident avec des changements importants dans l'offre programmatique des partis et, en particulier, $\mathrm{CiU}$ modifie son traditionnel positionnement quant aux relations Espagne-Catalogne pour défendre un programme indépendantiste et la tenue d'un référendum d'autodétermination. 
Lacarteélectoraleet parlementaire, àl'issue decesélections, apparait al ors commela pluséclatée de toutela période du gouvernement autonome ${ }_{1}^{17}$ avec une force politique ( $\mathrm{CiU})$ dont le score avoisine un tiers des suffrages exprimés, dépassant celui detousses rivaux sans que, pour autant, ceci lui permette deconstituer unemajoritéà elleseule et que, par ailleurs, on puisse également envisager de majorité sans elle.

En ce sens, les graphiques suivants rendent bien compte de certaines des disparités entre l'actuel système de partis actuel et celui de la fin des années 80 et du début des années 90. Dans chacun de ces graphiques se trouvent représentés le poids électoral des partis et les distancesidéologiques les séparant les uns des autres au sein de cet espace bidimensionnel que dessinel'intersection entre les deux axesà partir desquels se développela compétition électoraleen Catalogne : l'axe défini par la distinction gauche droite et celui formé par le rattachement à l'un ou à l'autre des deux pôles catalaniste ou espagnolisant.

Graphique 3. La compétition entre partis aux élections autonomiques de 1988

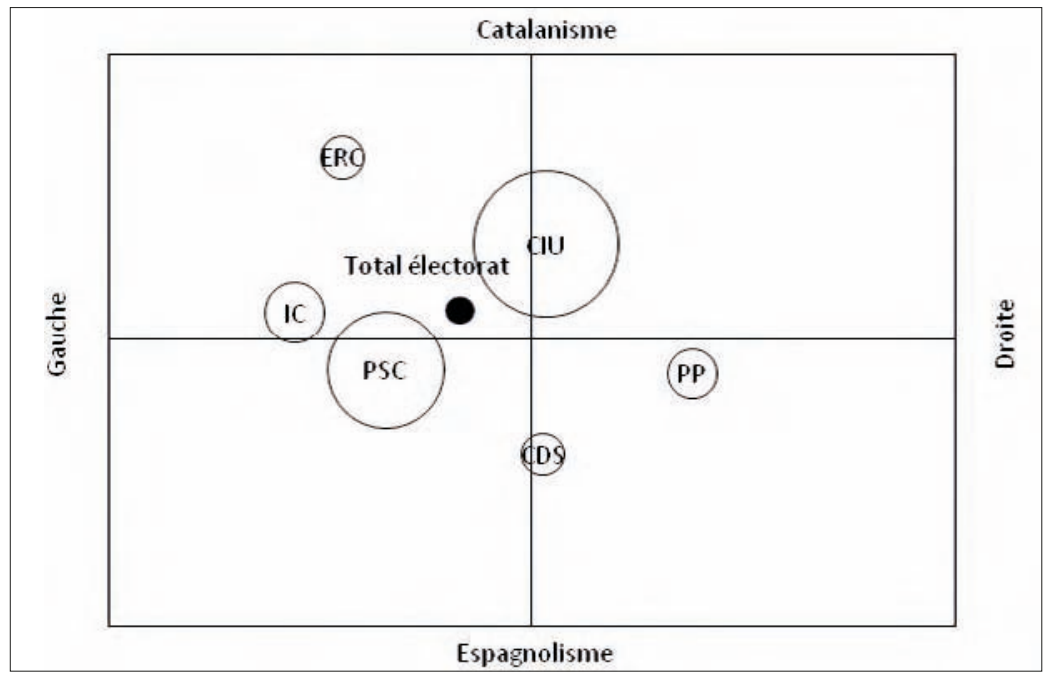

L'emplacement des partis sur cette carte résulte des interviews de personnes, reconnaissant avoir voté pour l'un d'entre eux lors des élections autonomiques de 1988, qui ont répondu aux questions concernant leur positionnement sur l'échelle gauche-droite et leur sentiement d'appartenance par rapport à la Catalogne et à l'Espagne. La taille différente de chacun des cercles représentant les partis indique en comparaison leur poids électoral respectif. Le point noir représente la position de l'électorat dans son ensemble.

Source : Sondage d'opinion sur la Catalogne réalisé par l'ICPS en 1991.

17. Les résultats électoraux attestent de la faiblesse des deux grands partis du système, à savoir CiU et plus particulièrement le PSC, constat à mettre en perspective avec le vieillissement de leur électorat respectif : plus delamoitié desélecteurs de 2012 nejouissaient pasdu droit devote lors des premières élections démocratiques. Concernant les autres formations politiques, C's connaît, en termes relatifs, l'ascension électorale la plus importante de ces élections (obtenant 9 députés) ; ERC multiplie par deux le nombre de ses suffrages, retrouvant son niveau de 2006 et la CUP, en engrangeant 3,50\% des votes exprimés, accède au Parlement tandis que ICV et le PP améliorent leurs résultats. 
Le premier graphique montre le système des partis tel qu'il se manifeste à partir des élections autonomiques de 1988 lorsque six partis obtiennent une représentation parlementaire avec cependant deux d'entre eux engrangeant la plupart des suffrages, CiU et le PSC, augurant une sorte de bipartismeasymétrique dans lequel le développement dela compétition électorale tourne au profit en particulier de CiU. Au cours de cette étape du système de partis, socialistes et partis sympathisants se trouvent en positions privilégiées pour obtenir une large partie des suffrages. À l'inverse, ERC et le PP, sur des positionnements extrêmes et opposés entre eux, se trouvent éloignés de l'épicentre dela compétition électorale, représenté par la position médianede l'ensemble de l'électorat, tandis que la situation du Centro D emocrático y Social (CDS), en voie de disparition, s'avère très fragile, également loin du cœur d'un électorat caractérisépar une sensibilitéde gauche et un catalanisme, tous deux modérés.

Cette carte de la compétition électorale se caractérise par le nombre de forces politiques en présence, le bipartisme asymétrique CiU et PSC ainsi que par une déconcentration du vote par rapport à ce qui se produit lors des élections nationales. En outre, elle se distingue aussi par de faibles différences entre partis, au-delà de ce que I'on constate entre socialistes et IC, s'agissant delagauche, et naturellement il en vademême pour l'opposition PSC et CiU dont les résultats, pour une large partie, finissent par se régler sur d'autres arènes politiques, accompagnés par le phénomène de vote dichotomique et l'abstention différentielle, évoqués précédemment.

Graphique 4. La compétition entre partis aux élections autonomiques de 2012

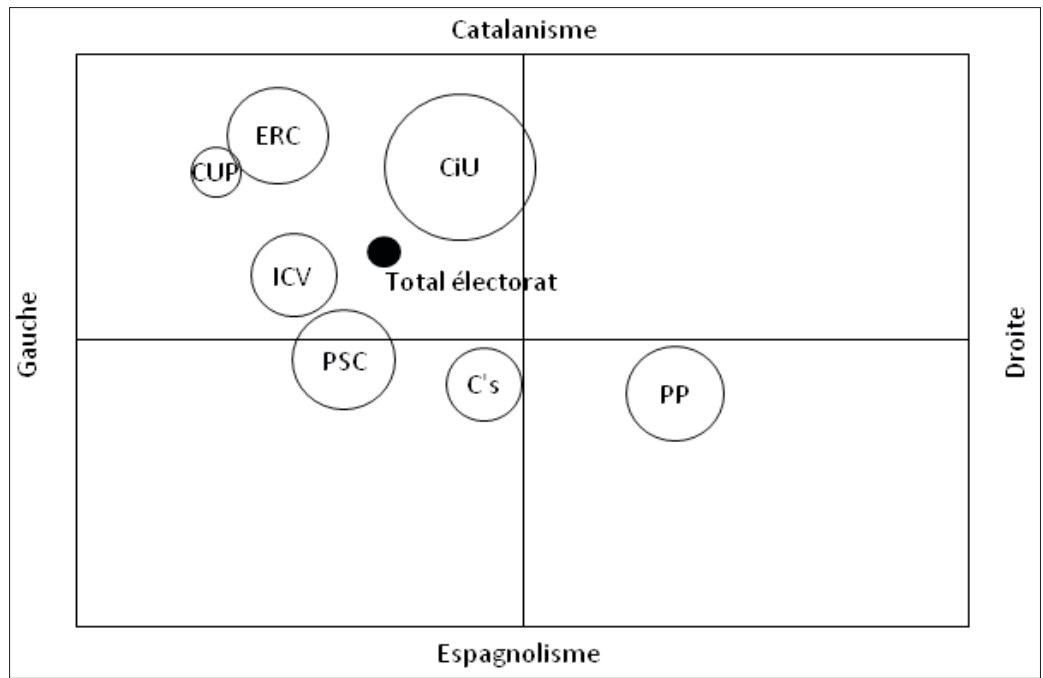

L'emplacement des partis sur cette carte résulte des interviews de personnes, reconnaissant avoir voté pour l'un d'entre eux lors des élections autonomiques de 2012, qui ont répondu aux questions concernant leur positionnement sur l'échelle gauche-droite et leur sentiement d'appartenance par rapport à la Catalogne et à l'Espagne. La taille différente de chacun des cercles représentant les partis indique en comparaison leur poids électoral respectif. Le point noir représente la position de l'électorat dans son ensemble.

Source : Sondage d'opinion sur la Catalogne réalisé par l'ICPS en 2012. 
Par contre sur le graphique qui représente la configuration du système de partis et la concurrence électorale, à partir des élections autonomiques de 2012, on observe une série de nouveautés importantes. En premier lieu, le centre de gravité de l'électorat et de l'ensemble des partis, en survolant le cadre supérieur gauche du graphique, se déplace vers des positions plus à gauche et davantage catalanistes. En deuxième lieu le nombre de forces politiques s'accroit tout comme le niveau de fractionnement du système. La déconcentration du vote s'accentue et contribue à façonner un Parlement au sein duquel une force politique, CiU, jouit dela majorité des sièges tandis que les autres partis se répartissent le restant dans des proportions, sinon identiques, non plusextrêmement inégales. II s'agirait d'un système dit d'« un partido y muchos » dans lequel C iU se transforme en force nécessaire, mais non suffisante, pour constituer des majorités parlementaires.

En troisièmelieu, peut-êtrel'un des aspects les plus importants de ce nouveau scénario, la polarisation idéologique du systeme s'accentue considérablement. Bien qu'on enregistre un glissement important de l'ensemble des partis vers la gauche et le nationalisme catalan ${ }^{18}$, la distance s'accroit également entre eux. Si la polarisation gauche-droite était en 1988 de 1,54 points et celle correspondanteà l'axe identitaire de 1,57 points, en 2012 toutes deux progressent allant jusqu'à atteindre respectivement 2, 15 et 3, 29 points. Ce qui revient à dire que la polarisation gauche-droite progresse de $40 \%$ tandis que celle relative au nationalisme atteint $109 \%$ d'augmentation. ${ }^{19}$

En quatrième et dernier lieu, la combinaison d'une fragmentation politique, plus importante, ajoutée à la déconcentration du vote et à l'augmentation de la polarisation favorise la prolifération de nouvelles aires de concurrence. Ainsi ERC et CiU s'affrontent pour le vote nationaliste catalan ; ERC, ICV et la CUP rivalisent dans la mouvance catalaniste et la gauche; le PSC et ICV se disputent surtout les faveurs de la gauche modérée; les socialistes sont susceptibles de perdre des soutiens à plusieurs niveaux ( CiU , ERC, ICV , C's) ; et lePP, CiU et C's tentent de s'approprier l'électorat plus modéré.

La multiplicité de domaines litigieux et conflictuels rend insaisissable la concurrence entre partis dont les resultats se trouvent alors beaucoup plus difficiles à prévoir dans la mesure où ils dépendent beaucoup plus des flottements dans le choix des électeurs et des stratégies des différentes acteurs publics à chaque instant.

18. LePP qui graviteautour de positions plus hispaniques sesépare de cemodèle.

19. Ces chiffres procèdent de l'adaptation de la mesure de polarisation proposée par Hazan (1995, 1997, pp. 118-19) qui en l'espèce a été calculée par rapport à chacun des axes de compétition. 


\section{UN TERRITOIRE, DEUX ÉLECTIONS... ET UN THÈME : LES RELATIONS ENTRE LA CATALOGNE ET L'ES- PAGNE}

S'il y a une question qui a marquéla politique catalanec'est sans nul doute celle des relations entre la C atalogneet l'Espagne. II n'est pas abusif d'avancer que la décentralisation politique en Espagne répond pour une large mesureà la volonté de ménager la C atalogne dans I'Espagne. II s'agit d'une constante de la politique espagnole actuelle. À partir de 1898 quand l'Espagne perd ses dernières positions coloniales, le catalanisme choisit de construire un projet politiquequi passe par l'instauration d'institutions degouvernance autonome pour la $\mathrm{C}$ atalogne. C e projet franchira une première étape avec la création de la M ancomunitat (Communauté) de Catalogneen 1914 (union en une seule institution des quatre Députations provinciales de $C$ atalogne) et une deuxième étape avec l'instauration de la Généralité sous l'époque républicaine (1931) et des électionsau Parlement de Catalogne en 1932.

Le franquisme n'a pas édulcoré la problématique. De fait, la dénommée « question régionale » fut alors l'une des questions clé dans la transition vers la démocratie après la période franquiste, problématique au travers de laquelle la place delaC atalogne jouerait un rôleessentiel. En fait, avant même l'adoption de la C onstitution espagnole, la G énéralité de C atalogne a été restaurée en janvier 1977 dans l'unique décision constituante prise al ors que les nouvelles institutions démocratiques ne s'étaient pas affrontées sur la réforme de la légalité franquiste mais sur la légalité de l'étape républicaine.

Les élections fondatrices de 1980 auguraient l'autogouvernement démocratique contemporain conduit par les gouvernements de CiU présidés par Jordi Pujol. Au cours de cette période de plus de trente ans, la question de la place de la $\mathrm{C}$ atalogne en Espagne est demeurée toujours présente quoique le contenu concret de la problématique, son importance, et sa traduction en termes de choix préférés des citoyens pour l'une ou l'autre des formes de relations entre Espagne et C atalogne s'est poursuivie en changeant au gré du processus politique. En fait on peut distinguer diverses étapes au cours de ce processus. La première étape serait caractérisée par le développement, la construction et le déploiement des nouvelles institutions d'autogouvernement. II s'agit d'une séquence animée par les gouvernements socialistes dirigés par Felipe González en Espagne et les gouvernements de Jordi Pujol en Catalogne, englobant les annees 80 et le début des années 90 . Au cours de cette période la question catalane occupa moins de place dans l'agenda politique qu'elle n'en aura ultérieurement : la virulence du terrorisme de l'ET A, la stabilité politique des majorités socialistes et les questions centrales qui ont à voir avec l'essor des institutions démocratiques et l'État providence permirent alors d'établir un débat plus apaisé sur la question territoriale.

À partir des années 90 la question territoriale va devenir l'un des points majeurs de l'agenda politique. Á ce moment là, l'État des Autonomies était parvenu à un carrefour à partir duquel il était désormais possible aux $\mathrm{Com}$ - 
munautés Autonomes de la dénommée « vía lenta » de disposer du droit de redéfinir leur autogouvernement et de le rendre équivalent à celui de la Catalogne, du Pays basque, de la Galice et de l'Andalousie ${ }^{20}$. Ce débat, qui déboucha sur les Pactes Autonomiques de 1992 entrelePS et lePP, encouragea les demandes du gouvernement de Catalogne afin que soient respectées les spécificités et renforcé l'autogouvernement catalan. La question catalane occupera encore davantage de place lorsque, en l'absence de majorités parlementaires au Parlement espagnol, CiU parviendra à être l'allié parlementaire du dernier gouvernement socialiste présidé par Felipe G onzález (19931996) et ensuite du premier gouvernement PP préside par José M. Aznar (1996-2000).

La question va se polariser définitivement avec le nouveau millénaire. La volonté du gouvernement de C atalogne d'aller plus en avant dans l'autogouvernement, ajoutée au fait que le gouvernement PP de José M aria Aznar souhaite de son côté une recentralisation (après qu'il ait obtenu la majorité absolue aux élections générales en 2000), va accroître la demande des partis catalans en faveur d'un dépassement de l'actuel Statut d'Autonomie de 1979. Le changement de gouvernement en C atalogne en 2003 (avec l'arrivée du gouvernement de coalition de gauche dirigé par le socialiste Pasqual $\mathrm{M}$ aragall) setraduit par la volonté d'élaborer un nouveau Statut, approuvé d'abord par le Parlement catalan, accepté après modification par le Parlement espagnol (avec le soutien corollaire du Parti socialiste) et ratifié définitivement par referendum avec $73,90 \%$ de votes favorables et $48,90 \%$ de participation en Catalogne. Cependant le PP fit appel à l'encontre de cette nouvelle loi devant leT ribunal Constitutionnel qui décida d'en suspendre unelargepartieen juin 2010 après une procédure longue et tumultueuse. Cette situation eut comme effet de mobiliser une partie de l'opinion publique catalane en faveur de l'indépendance. Les mobilisations populaires prirent un tournant ouvertement indépendantiste en 2012 et en 2013, durant la Diada N acional deCatalunya.

Dans quelle mesure ont changé les choix préférentiels des catalans quant à leurs relations avec l'Espagne ? O n peut observer l'évolution de ces préfé rences sur le $5^{e}$ graphique dont les données relèvent des enquêtes de l'« Institut deCiènciesP olítiquesi Socials», sourcela plus ancienneet la plus amplesur cette question. L'indicateur utilisé a conduit à faire choisir les catalans à partir de 1991 entre quatre types de relations entre C atalogne et Espagne: la Catalogne comme région d'Espagne, comme Communauté Autonome, comme État fédéré et comme État indépendant. Les données mettent en évidence le fait que, bien qu'avec des fluctuations, le choix prédominant des catalans a été jusqu'à récemment le maintien du statu quo, à savoir laC atalogne en tant que Communauté Autonome de l'Espagne. Il est certain qu'au cours des années

$\overline{20 .}$ L'article 143 dela Constitution espagnole prévoyait la possibilitéd'accéder àun autogouvernement limité aux territoires qui le désirent sans qu'il ait nécessité pour cela de soumettre cette nouvelle organisation à référendum populaire. La plupart des 17 Communautés Autonomes espagnoles ont accédé à l'autogouvernement en empruntant cette voie. La Constitution pré voyait également que ces mêmes territoires pouvaient également accéder au niveau de l'autogouvernement tel qu'il avait existé dansles « CommunautésAutonomes historiques» , cinq ans après l'adoption de leurs Statuts respectifs. 
durant lesquelles fut négociéle nouveau Statut, les partisans du modèle fédéral ont vu leurs effectifs croitre quoique la mouvance autonomiste continua d'êtrece temps là l'option recueillant le plus de partisans.

C ependant, coïncidant avec la sentence du T ribunal Constitutionnel et la recrudescencedela criseéconomiqueen Espagne, deuxtendancessont apparues manifestes : la perte de soutien pour l'état actuel des choses et la forte augmentation (en très peu de temps) des partisans de l'indépendance. De fait, actuellement, l'indépendance est l'option choisie par le plus de citoyens avec $42 \%$ de réponses tandis que le maintien de la situation actuelle ( $\mathrm{LaC}$ atalogne, communauté autonome d'Espagne) continue de perdre des soutiens, parvenant à rester l'option préférée que d'un quart seulement des citoyens. D'un autre côté, les soutiens aux options fédéraliste et régionaliste n'enregistrent pas de grands changements ces temps derniers, recueillant respectivement $18 \%$ et $6 \%$ des choix exprimés.

Graphique 5. Evolution des préférences quant aux relations Catalogne Espagne

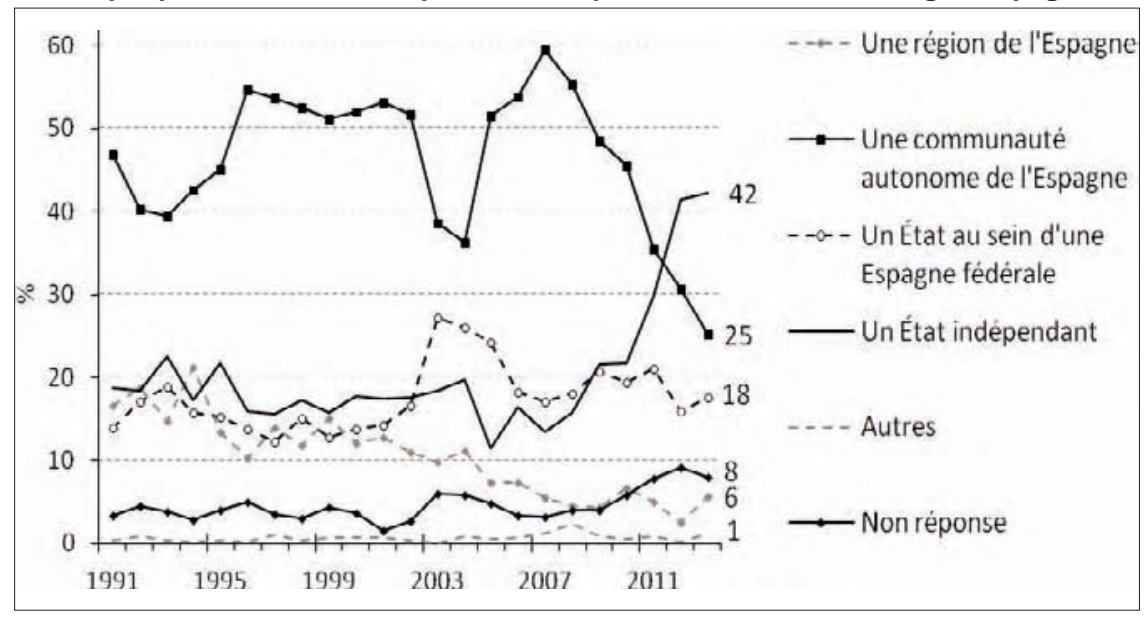

Source : Sondages d'opinion, Catalogne, ICPS

Devant cette répartition des préférences, qu'adviendrat-il en cas de referendum? Le 6 e graphique fait ressortir le fait qu'actuellement $49 \%$ des interviewés voteraient oui àl'indépendance, $25 \%$ non, $21 \%$ s'abstiendraient et $5 \%$ voteraient blanc, nul ou préfereraient ne pas se prononcer. Del'évolution des intentions de vote on peut distinguer deux éléments. D'un côté, aussi bien l'indicateur de préférences que l'intention de vote témoigne d'un soutien similaireà l'option indépendantiste. II n'en a pas toujoursété ainsi. En 2010 les soutiens à l'option indépendantiste atteignirent respectivement 30\% et $44 \%$. Par contre en 2012, juste après les élections autonomiques, les différences se réduisent considérablement ( 41 vs. 49) et se maintiennent actuellement ( 42 Vs. 49). Ceci signifie que le fait que CiU se soit engagée devant les citoyens à organiser une consultation lors de la dernière campagne électorale a provoquéune réaction dansl'opinion publique, l'amenantà prendreen plus grande 
considération cette question et à se montrer plus « congruente » dans ses réponses. D'un autre côté, il faut souligner qu'au cours de l'année 2013 les positions se sont cristallisées. En fait on enregistre uniquement une certaine augmentation des abstentionnistes et une certaine chute du vote non. En fin de compte, le fait que les inconnues sur les questions : comment et quand organiser la consultation, n'aient pas été résolues un an après le déroulement des élections, s'est traduit par peu de changements sur la répartition des préférences et les intentions de vote.

Graphique 6. Intention de vote à un referendum sur l'indépendance de la Catalogne

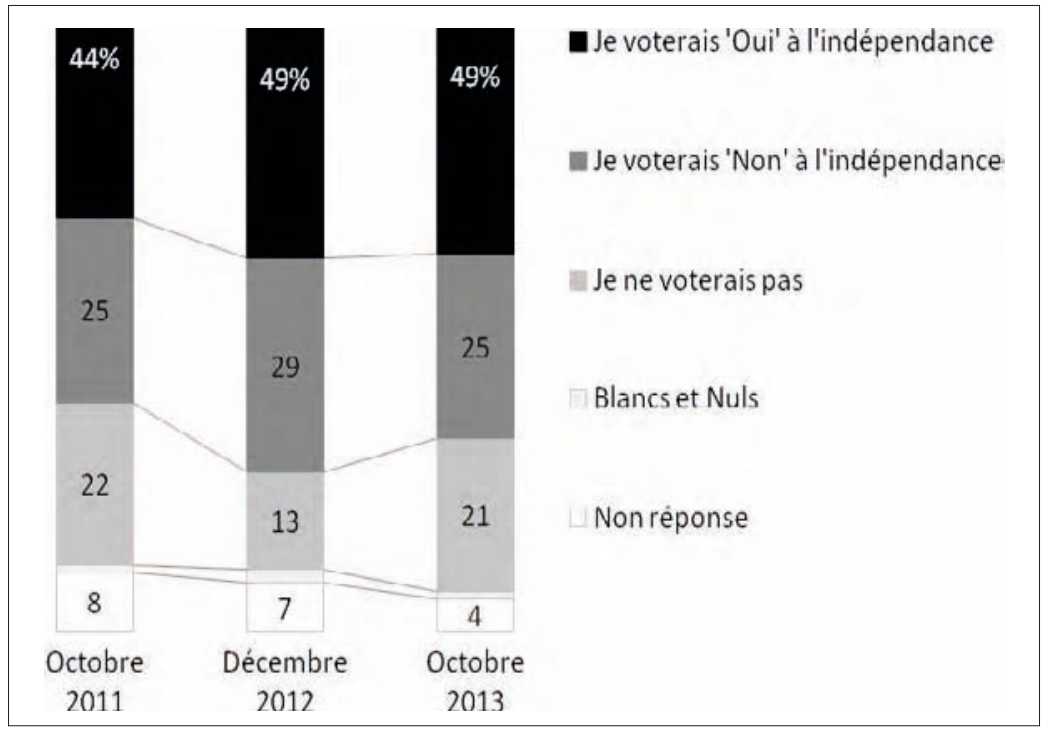

Source : Sondage d'opinion, Catalogne, réalisé par l'ICPS, 2013

\section{RÉFÉRENCES / REFERENCES}

Bartomeus 0 \& M edinaL. (2011), « La competencia entre partidos: isíntomas de cambio?», in M arcet J.\& C asals X. ( eds), Partidosy elecciones en la Cataluña del siglo XXI, Barcelona, Institut de Ciències Polítiquesi Socials.

Blanco I. \& M as P. ( 2008), La desafecció política a Catalunya. Una mirada qualitativa, Barcelona, Fundació Jaume Bofill.

Castro C. (2011), Retrato electoral deC atalunya. Claves para comprender tres décadas deelecciones catalanas (1980-2010), Barcelona, Ed. Episteme.

Colomer J. M . (1991), «El equilibrio político inducido estructuralmente», Revista del Centro deEstudios Constitucionales, $n^{\circ} 8$, pp. 27-38.

Font J., Contreras J. \& Rico G. (1998), L'abstenció en les eleccions al Parlament de Catalunya, Barcelona, Ed. M editerránea. 
H azan R. Y. ( 1995), « Centre Parties and Systemic Polarization. An Exploration of Recent T rends in W estern Europe », Journal of Theoretical Politics, vol. 7, $n^{\circ} 4$, pp. 421-445.

H azan R. Y. (1997), CentreParties. Polarization and Competition in European Parliamentary D emocracies, Londres, C ontinuum.

Liñeira R. (2013), « Cataluña ante la consulta sobre la independencia. Participación, voto y motivaciones», Q uaderns del'ICPS, $n^{\circ} 4$.

M arcet J. (2011), " Las relaciones entre el sistema de partidos de Cataluña y de España y la articulación partidaria », in M arcet]. \& C asals X. (eds), Partidos y elecciones en la Cataluña del siglo XXI, Barcelona, Institut de Ciències Polítiques i Socials.

M ontero J. R. \& Font J. ( 1989) , El voto dual en Cataluña: dimensiones, sujetos y factores, Barcelona, M imeo.

M ontero J. R. (1991) , « El voto dual: lealtad y transferencia de votos en las elecciones autonómicas », Estudis Electorals, vol. 10, pp. 183-211.

M olas I. \& Bartomeus O . (1988), « Estructura de la competencia política a C atalunya», W orking Papersn ${ }^{\circ} 138$, Barcelona, Institut de C iències Polítiquesi Socials.

M olas I. \& Bartomeus 0. ( 1999), " Els espais de frontera entre els electorats », W orking Papers 165, Barcelona, Institut de C iències Polítiques i Socials.

M uñoz] ., Guinjoan M . \& Vilaregut R. (2010), « Lesconsultes sobrela independencia: context, organització i participació » , Barcelona, Institut de Govern i Polítiques Públiques.

Pallares F. \& Font J. (1995), « Las elecciones autonómicas en Cataluña 19801992 », in Del Castilllo P. (ed.), Comportamiento político y electoral, M adrid, Centro de Investigaciones Sociológicas.

Riba C. (1995), Vot dual i abstenció diferencial. T resaproximacions a l'estudi del comportament electoral a Catalunya ( 1982-1993) , T esisD octoral, Bellaterra, U niversitat Autònoma de Barcelona.

Riba C. (2000), « Voto dual y abstención diferencial. Un estudio sobre el comportamiento electoral en Cataluña », Revista Española de Investigaciones Sociológicas, $n^{\circ} 91$, pp. 59-88.

Thomassen J. (2005), The European voter. A comparative study of modern democracies, 0 xford, 0 xford U niversity Press.

Torcal M . \& M edina L. (2007), « La competencia electoral entre PSOE y PP: el peso de los anclajes de ideología, religión y clase », in M ontero J. R., L ago I. \& Torcal M. (eds) , Elecciones Generales 2004, M adrid, C entro de Investigaciones Sociológicas.

Vallès J. M . (2008) , Actituds polítiques i comportament electoral a Catalunya: mate rials per a un debat social, Barcelona, G eneralitat de C atalunya.

Vallès). M. (2009), « La abstención diferencial: una nota sobre los casos de Catalunya y la Comunidad Autónoma deM adrid », Revista Española deC iencia Política, $n^{\circ} 21$, pp. 93-105. 